\title{
Intramedullary spinal cord abscess
}

\author{
M E Miranda Carús, B Anciones, A Castro, M Lara, A Isla
}

\begin{abstract}
We report a patient with a chronic intramedullary spinal cord abscess who suffered an episode of acute meningitis due to rupture of the abscess into the subarachnoid space.
\end{abstract}

Intramedullary spinal cord abscesses are rare; to date only about 65 cases have been described. ${ }^{1}$ We report a case of intramedullary thoracic spinal cord abscess. To our knowledge this is the first case in which an episode of acute meningitis may be associated with the rupture of an intramedullary abscess into the subarachnoid space.

\section{Case report}

A 61 year old man was admitted to the hospital because of impaired gait. $\mathrm{He}$ was well until three months earlier, when he noticed pain and paresthesia in the sole of his left foot and intermittent moderate back pain. Over the next two months, paresthesia extended to his left leg and thigh and the sole of his right foot. The patient consulted a neurologist, who reported no motor deficit but did observe an L5 left hypoesthesia and a decreased left ankle jerk. Lasegue's sign was negative bilaterally. Over the next 30 days he developed unstable gait with loss of strength and numbness in both legs as well as voiding urgency, bladder tenesmus, faecal incontinence, and impotentia coeundi. He was then referred to us by his neurologist for hospital admittance. He had psoriasis and a one year history of non-insulin dependent diabetes mellitus controlled by diet. He did not smoke and his alcohol intake was equivalent to $80 \mathrm{~g}$ a day.

General examination was normal except for the presence of urinary retention. He had no fever. On neurological examination we found a paretic gait, clear loss of strength in both legs (proximal and distal), decreased sensitivity under L2 level (tactile, nocioceptive, and proprioceptive), and saddle hypoesthesia. Anal sphincter tone was decreased. Both ankle jerks were abolished; left knee jerk was normal and right knee jerk was increased. Peripheral blood count and plasma biochemistry were normal except for a high glucose concentration $(280$ $\mathrm{g} / \mathrm{l}$ ). Serological markers for syphillis were negative. A dorsolumbar simple $x$ ray picture and a dorsolumbar myelography (metrizamide) performed on the first hospital day showed no alterations. Brain CT scan was normal. Elec- tromyography disclosed signs of a mild peripheral polyneuropathy with decreased nerve conduction speed (motor, sensitive, and mixed) as well as signs of moderate muscle denervation in the legs.

Neurological deficit progressed during hospitalisation. On the 10th hospital day neurological examination showed a complete loss of strength and sensation in both legs, a motor deficit in the lower spinal muscles, and a complete D12 sensitive deficit (tactile, nociceptive, and proprioceptive). A spinal MRI disclosed no abnormalities. On the 20th hospital day he developed high fever, chills, cephalea, and neck stiffness. A lumbar puncture showed increased CSF pressure and a purulent CSF with leucocytes $5.0 \times 10^{9} / 1(95 \%$ poly-

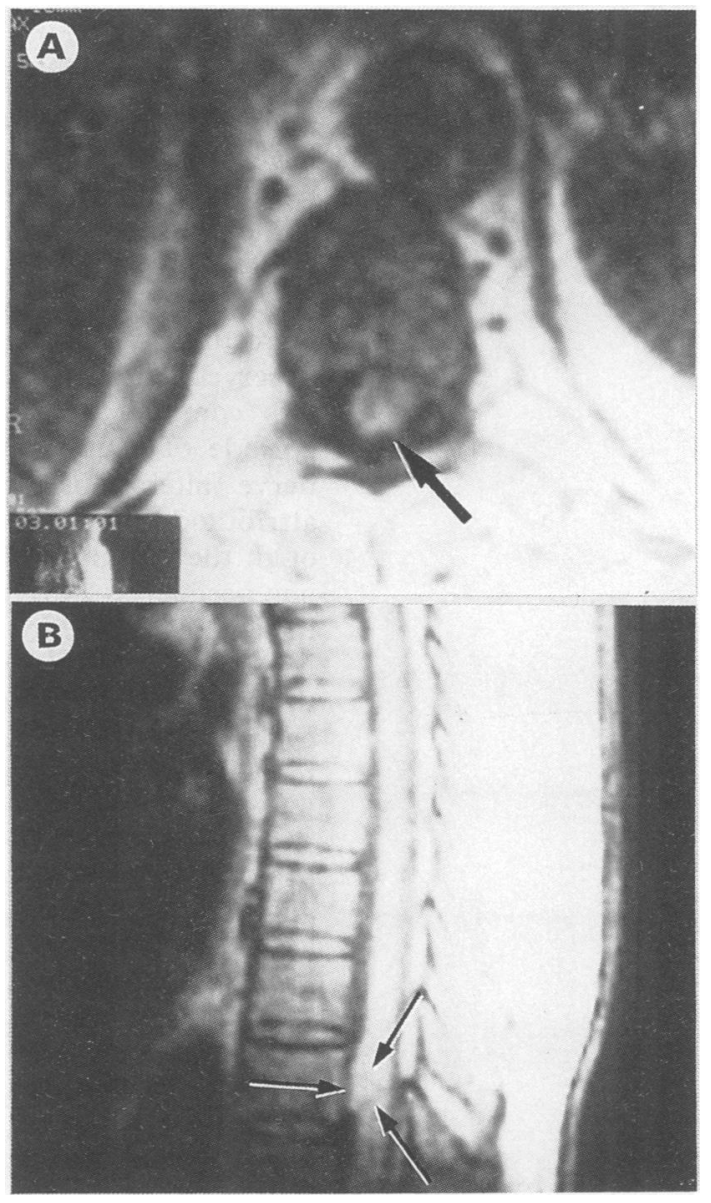

Figure (a) Saggital, paramedian, T-2-weighted dorsal $M R I$ demonstrating isodense anterior lesion at $D 9$ level (arrows). Lesion is poorly distinguished because it produces T-2 signal similar to that of normal parenchyma. (b) Axial T-1-weighted, dorsal spine MRI section at $D 9$ level, showing anterior placed abscess (arrow) 
morphonuclear), protein $705 \mathrm{mg} / \mathrm{l}$, glucose 510 $\mathrm{mg} / \mathrm{l}$ (glucaemia $251 \mathrm{mg} / \mathrm{l}$ ), and chloride 110 $\mathrm{mmol} / \mathrm{l}$. He was treated with parenteral cefotaxime and recovered uneventfully. Microbiological studies of blood and CSF were negative. On the 25th hospital day, a second MRI of the dorsolumbar spine showed a hypoisodense intramedullary round image on the left anterior part of the spinal medulla at D9 level (figure). Intravenous treatment with dexamethasone was begun, and a D7-10 laminectomy was performed on the 26th day. No abnormalities of the dural or arachnoidal spaces were found on operation. The medulla was not enlarged and was normal in appearance except for a tiny recent scar on the left anterior spinal surface at D10 level. After slight manual compression the scar opened and about $1.5 \mathrm{ml}$ pearly-grey fluid spontaneously drained from an encapsulated area within the parenchyma. After operation, the patient was treated with dexamethasone and antibiotics. Histopathological examination demonstrated a fibrous capsule and a non-specific abscess. Microbiological studies of the operative specimen were all negative. After surgery the patient underwent physiotherapy, recovering some strength in his left leg muscles, but remained paraparetic. One year after the operation paraparesis, urinary retention, and faecal incontinence persisted.

\section{Comment}

The patient had an intramedullary spinal cord abscess located at D9 level. The abscess may have been of primary origin ("spontaneous") because no source of infection was apparent on clinical history and physical examination. It followed a subacute clinical course with a space-occupying clinical expression. No signs of central nervous system infection were observed until a late stage, when paraparesis was complete, and the patient suffered an episode of acute meningitis. The peripheral nerve alterations seen on EMG may be attributed to a diabetic polyneuroathy. Although the initial MRI was normal, the second one performed 15 days later demonstrated the intramedullary lesion. The failure of the first MRI to reveal the lesion shows the evolution of the pathological process. At the time of initial evaluation, the lesion probably consisted of a focus of medullitis without necrosis or pus formation that was beyond the resolution of the MRI scan. Enlargement of the lesion and progression to abscess formation subsequently allowed its detection. A time course of one month would be consistent with the evolution of this pathological process.

Intramedullary spinal cord abscesses (ISCA) are uncommon. To date only 64 cases have been described. ${ }^{1}$ Thirty patients with ISCA have been operated on, with six deaths, five occuring in the days before antibiotics. Among the survivors, several cases with a good functional recovery after operation have been reported. ${ }^{2-6}$ The return of function in these cases is in clear contrast to that of epidural spinal abscesses, in which a good functional recovery is almost unknown once conduction in the spinal cord has become seriously affected. ${ }^{1}$ Presumably, the centrally placed abscess is less likely than the epidural abscess to produce irreversible vascular changes in the cord. Despite other chronic ISCA patients having a successful functional outcome ${ }^{27}$ our patient showed only a slight functional recovery, probably due to the long duration of symptoms before diagnosis was established. Staphylococcus and streptococcus are the organisms most often cultured from ISCA. When all age groups are considered and modern bacteriologic methods are used, however, anaerobic and microaerophilic organisms collectively are the most common cause. Among the reported cases of ISCA, staphylococcus was identified in 14, streptococcus in 10, gram-negative pathogens in six, multiple organisms in six, actinomyces in three, and pneumococcus and listeria in one each. Proteus species were cultured in two ISCA associated with a dermal sinus. In 23 patients ( $36 \%$ ), as in our case, no organism was identified by culture. Patients with subacute and chronic spinal cord abscesses present with a stuttering neurological deterioration, and the symptoms and signs may mimic those of an intramedullary tumour. ${ }^{1-8}$ To our knowledge no case of acute meningitis has been described in the course of a chronic or subacute spinal cord abscess after the development of neurological deficit.

Recurrent bouts of meningitis with the sudden onset of transverse myelitis sould raise the suspicion of a congenital dermal sinus with an intramedullary abscess, ${ }^{8}$ In our patient, however, a single episode of meningitis was observed, no anatomical defect could be demonstrated on physical examination or myelography, and a complete paraparesis was present at onset of meningitis. We believe that in this case meningitis may have been secondary to the rupture of the ISCA into the subarachnoidal space. The absence of any other apparent focus of infection, the negativity of blood cultures, and the finding of a thin layer of highly vascular, friable, scar tissue, separating the abscess from the subarachnoid space are all clues that support this hypothesis.

We thank Dr J Uson for help in preparing the manuscript.

1 Menezes AH, Van Gilder JC. Spinal cord abscess. In: Wilkins RH, Rengachari SS, eds. Neurosurgery (vol III) New York: McGraw-Hill, 1985:1969-72.

2 Manfredi M, Bozzao L, Frasconi F. Chronic intramedullary abscess of the spinal cord. $J$ Neurosurg 1970;33:352-5.

3 Morrison RE, Brown J, Gooding RS. Spinal cord abscess caused by Listeria monocytogenes. Arch Neurol $1980 ; 37: 243-4$.

4 Maurice-Williams RS, Pamphilon D, Coackham HB. Intramedullary abscess, a rare complication of spinal dysraphism. J Neurol Neurosurg Psychiatry 1980;43: 1045-8.

5 Fortuna A, Contratti F, Di Lorenzo N. Cervical intramedullary abscess; extirpation by means of microsurgical techniques. J Neurosurg Sci 1979;23:159-62.

6 Bean JR, Walsh JW, Blacker HM. Cervical dermal sinus and in J Walsh JW, Blacker HM. Cervical dermal sinus an intramedullary sping $1979 ; 5: 60-2$

7 Walker RM, Dyke SC. Abscess of the spinal cord. Lancet 1936;1:1413-4.

8 D'Angelo CM, Whisler WW. Bacterial infections of the spinal cord and its coverings. In: Vinken PJ, Bruyn GW, eds. Handbook of clinical neurology (vol 33). Amsterdam: Elsevier/North Holland Biomedical Press, 1978:187-94. 